\section{WEB WATCH}

\section{Centre of the stroke}

Hippocrates, the father of medicine, was the first to identify the sudden onset of paralysis more than two millennia ago. As its specific origin was not known, the general term 'apoplexy' was coined to describe this condition. It was only in the seventeenth century that postmortem analysis began to show that bleeding in the brain was one of the underlying causes for the paralysis, giving rise to our modern concept of stroke as a cerebrovascular disease.

In spite of this long tradition, we still have a limited battery of tools to treat stroke and prevention is arguably our best strategy to keep this devastating condition at bay. Indeed, the large number of educational campaigns on what stroke is and how to reduce any risk factors attest to the importance of receiving reliable information to cope with this condition. If you are looking for information on stroke, then you must visit the Internet Stroke Center web site, a comprehensive source of professional information about cerebrovascular disease.

The Internet Stroke Center is a non-profit, purely educational web site from Washington University in St Louis directed by Mark P.

Goldberg. The web site editor is Adam Istas and the content is supplied by experts at the (clinical) Stroke Center at Washington University and around the world. The site includes the only

continuously updated database of clinical trials and research agents (the Stroke Trials Directory), with more than 400 detailed listings. The site also offers extensive directories of stroke publications, syndromes, treatment centres and conferences. In addition, it contains more than 20 detailed, referenced teaching presentations, which constitute a remarkable educational asset.

Juan Carlos López
This resulted in unbalanced retinal activity for 4-14 days during what was now established as the early stage of column establishment. It was found that normal column structures were still formed in these animals, and both eyes were still equally represented in the cortex.

The authors concluded that the initial establishment of the ocular dominance columns is able to pro- ceed independently of visual stimulus from the retina. Changes in retinal activity during the critical period are therefore likely to refine or disrupt an existing pattern, rather than establish a new one. This work opens up a new field of investigation to identify the intrinsic molecular guidance cues that are required for the patterning of the visual cortex.
(Q) References and links ORIGinal Research PAPER Crowley, J. C. \& Katz, L. C. Early development of ocular dominance columns. Science 290, 1321-1324 (2000)

FURTHER READING Crowley, J.C \& Katz, L. C. Development of ocular dominance columns in the absence of retinal input. Nature Neurosci. 2 , 1125-1130 (1999) | Shatz, C. J. Emergence of order in visual system development. Proc. Natl Acad. Sci. 93, 602-608 (1996) WEB SITE Lawrence C. Katz ENCYCLOPEDIA OF LIFE SCIENCES VISUal system development in vertebrates

\title{
MOTOR CONTROL
}

\section{Mind over matter}

All her thoughts focused on the glass, willing it to move. Slowly, its base lifted off the table. Resting on one edge, the glass wobbled briefly before toppling over, spilling its contents - water plus newt - all over the evil headmistress. This scene, from Roald Dahl's classic children's book Matilda, is an example of a popular literary theme - being able to control objects by the 'power of thought'.

The idea, however, is leaving the realms of fiction. Reporting in Nature, Wessberg and colleagues describe how electrical signals from a monkey's brain instructing arm movement can be used to move robotic arms in an identical manner. Their research offers new hope for efforts aimed at allowing paralysed people to control artificial limbs by brain activity alone.

The initial challenge in developing an artificial limb control system is how to extract complex movement information from the activities of large populations of neurons in real time. Building on two decades of studies linking several areas of the cerebral cortex with the control of arm movements, Wessberg et al. now provide an answer to this problem.

First, neuronal signals detected by microelectrodes implanted in several regions of the cerebral cortex of owl monkeys were simultaneously monitored while the monkeys were trained in arm movement tasks. Coherence analysis revealed that the activities of most single neurons in each of the recorded cortical areas were significantly correlated with hand trajectory, thus allowing each neuron to be assigned a coefficient related to its role in the task. Computer algorithms based on these coefficients were then used to predict hand position in real time, and proved to be highly successful, despite the complexity and variability of the movement. Accurate hand trajectory predictions were obtained quickly and remained stable for long periods of time. Consequently, Wessberg et al. were able to use brain-derived signals to control the movements of robotic arms both locally and over the internet, with the computer program determining the new position of the arms at each instant on the basis of neuronal signals collected in the past second. The experiments also investigated the contributions of different cortical regions to the predicted movement. By calculating the average effect of removing single neurons one by one from the observed population, it was shown that neurons in the dorsal premotor cortex provided the highest contribution, with those in the posterior parietal cortex and primary motor cortex providing comparably lower contributions. So, such systems should be useful for elucidating which regions of the brain control which features of limb movement. Hopefully, further advances in understanding combined with extensions of the techniques described will one day, as the authors suggest, lead to "a

brain-machine interface for allowing paralysed patients to control voluntarily the movements of prosthetic limbs".

Peter Kirkpatrick

(2) References and links ORIGINAL RESEARCH PAPER Wessberg, J. et al. Real-time prediction of hand trajectory by ensembles of cortical neurons in primates. Nature 408, 361-365 (2000) FURTHER READING Mussa-Ivaldi, S. Real brains for real robots. Nature $\mathbf{4 0 8}$ 305-306 (2000) 